\title{
Source-tracking of the Chinese Chikungunya Viruses Suggests That Indian Subcontinent and Southeast Asia Act as Major Hubs for the Recent Global Spread of Chikungunya Virus
}

\section{Shaofu Qiu}

Chinese PLA Center for Disease Control and Prevention https://orcid.org/0000-0001-5070-9294 Jinpeng Guo

Chines PLA Center for Disease Control and Prevention

\section{Peihan Li}

Chinese PLA Center for Disease Control and Prevention

\section{Peng Li}

Chinese PLA Center for Disease Control and Prevention

\section{Xinying Du}

Chinese PLA Center for Disease control and Prevention

\section{Rongzhang Hao}

Chinese PLA Center for Disease Control and Prevention

\section{Chaojie Yang}

Chinese PLA Center for Disease Control and Prevention

\section{Qi Wang}

Chinese PLA Center for Disease Control and Prevention

\section{Hongbo Liu}

Chinese PLA Center for Disease Control and Prevention

\section{Haoran Zhang}

Chinese PLA Center for Disease Control and Prevention

\section{Sai Tian}

Chinese PLA Center for Disease Control and Prevention Hua Shi

Chinese PLA Center for Disease Control and Prevention Liang Wen

Chinese PLA Center for Disease Control and Prevention

\section{Daizhi An}

Chinese PLA Center for Disease Control and Prevention

\section{Xiaocui Yang}




\section{Xiaoyuan An}

Fourth People's Hospital of Zunyi

\section{Ligui Wang}

Chinese PLA Center for Disease Control and Prevention

\section{Hongbin Song}

Chinese PLA Center for Disease Control and Prevention

Changjun Wang ( $\square$ wangchj102@126.com )

Chinese PLA Center for Disease Control and Prevention

\section{Research Article}

Keywords: Chikungunya virus, whole genome sequencing, phylogenetic analysis, evolution, adaptive mutation

Posted Date: June 18th, 2021

DOI: https://doi.org/10.21203/rs.3.rs-613108/v1

License: (c) (i) This work is licensed under a Creative Commons Attribution 4.0 International License. Read Full License 


\section{Abstract}

\section{Background}

Chikungunya fever caused by Chikungunya virus (CHIKV) has become a major global health concern, which has caused unexpected large outbreaks in Africa, Asia, Europe, and the Americas. China is a nonindigenous region of $\mathrm{CHIKV}$, and the origin of the Chinese CHIKV strains is not well understood. Especially, there is limited understanding of the recent global spread of CHIKV in the context of recent worldwide epidemic of CHIKV.

\section{Methods}

Here we investigated a novel Chikungunya patient imported from Myanmar into China in August, 2019. The genome was sequenced directly from the serum sample by combined MinION sequencing with BGISEQ-500 sequencing. We constructed a complete CHIKV genome dataset, including 727 CHIKV genomes retrieved from Genbank and the genome sequenced in this study, and conducted an updated and comprehensive phylogenetic analysis to understand the origin, evolution, transmission routes and genetic adaptation of the virus.

\section{Results}

All of the globally distributed CHIKV genomes were divided into three genotypes, including West Africa (WA), East/Central/South African (ECSA) and Asian genotypes. The genome sequenced in this study was located in the Indian Ocean lineage (IOL), and closely related to a strain isolated from a patient in Australia who returned from Bangladesh in 2017. Comprehensive phylogenetic analysis showed that the Chinese strains were mainly sourced from Indian subcontinent and Southeast Asia. Further analyses indicated that Indian subcontinent and Southeast Asia may act as major hubs for the recent global spread of CHIKV, leading to widespread epidemics and even many large outbreaks. Moreover, we identified 179 distinct sites containing some undescribed sites in the SP and nsP proteins, showing obvious genetic varieties associated with different CHIKV lineages.

\section{Conclusions}

Here we reported a novel CHIKV isolate from a Chikungunya patient imported from Myanmar into China in 2019, and summarized the source and evolution of Chinese CHIKV strains. The present findings provided better understanding of the recent global evolution of CHIKV, highlighting the urgent need for the strengthened surveillance including the viral diversity surveillance.

\section{Introduction}

The Chikungunya virus (CHIKV) causes Chikungunya fever, a febrile illness with severe arthralgia and rash, and even severe clinical symptoms The Chikungunya virus (CHIKV) causes Chikungunya fever, a febrile illness with severe arthralgia and rash, and even severe clinical symptoms [1]. CHIKV is believed to 
have originated in Africa, and the first case of Chikungunya fever was recognized in an outbreak occurred in Tanzania in 1952 [2]. Since then, CHIKV has caused widespread epidemics and even many large outbreaks [1, 3]. Especially since 2004, CHIKV has caused unexpected large outbreaks in Africa, Asia, Europe, and the Americas, becoming a major global health concern $[3,4]$.

Uncovering the epidemiological patterns and changing trends of CHIKV plays a crucial role in its prevention and control. China is a non-indigenous region of CHIKV, and most cases were imported and mainly identified in Guangdong and Zhejiang Provinces of China $[2,4]$. Notably, there are also several local Chikungunya outbreaks which have been documented in these two Provinces, China [2, 4]. Here we identified a novel CHIKV isolate from a Chikungunya patient imported from Myanmar in August, 2019. The origin of the Chinese CHIKV strains is not well understood. Previous studies have described the origin, evolution and spread of CHIKV globally, however there is limited understanding of the recent global spread of CHIKV in the context of recent worldwide epidemic of CHIKV. Here we conducted an updated and comprehensive phylogenetic analysis by using a global genome dataset to understand the origin, evolution, transmission routes and genetic adaptation of the virus.

\section{Materials And Methods}

A 42-year-old male Chinese patient with fever, cough and fatigue was admitted to a hospital in Zunyi City, Guizhou Province, China on August 8, 2019. This patient was engaged in pepper planting and sales in Yangon, Myanmar this year, and had an abrupt fever at about 17 o'clock on August 7, 2019. He then flied back to Kunming City, Yunnan Province, China at 19 o'clock, and back to Zunyi City for emergency treatment at 6 o'clock on August 8, 2019. The blood samples were collected and sent to our lab for further microbiological detection. A multiplex quantitative reverse transcription-PCR (qRT-PCR) kit (Shenzhen Unimedica Co.,Ltd, Shenzhen, China) was used to screen seven pathogens including Chikungunya (CHIKV), Dengue (DENV), Japanese encephalitis (JEV), West Nile (WNV), Yellow fever (YFV), Sindbis (SINV) and Zika (ZIKV) viruses. After 11 days treatment, the patient was recovered and discharged from the hospital.

Whole-genome sequencing (WGS) was performed directly from the serum sample by using MinION and BGISEQ-500 platforms in our lab, respectively. De novo assemblies were generated using Canu v1.6 [5] for MinION sequencing and MEGAHIT V1.1.4 [6] for BGISEQ-500 sequencing. We constructed a complete CHIKV genome dataset, including 727 CHIKV genomes retrieved from Genbank (access date 11/01/2019) and the genome ZY1908 (accession no. MN756625) sequenced in this study (Table S1). A comprehensive phylogenetic analysis was performed to trace the origin of the Chinese strains and understand the origin, evolution, and transmission routes of the virus. In order to better understand the evolutionary influences on CHIKV genotypes, we conducted a thorough screening of the lineage-specific varieties in both structural and non-structural proteins (sP and nsP) based on the $728 \mathrm{CHIKV}$ sequences.

\section{Results}


Epidemiological investigation showed that this patient had been living in Yangon, Myanmar for nearly half a year, and had been bitten by mosquitoes on his calf before the onset of the disease. The genome was rapidly and directly obtained from the serum sample by combined MinION sequencing with BGISEQ500 sequencing (Fig. 1). Here we obtained 29 Chinese CHIKV genomes (Table S2). Phylogenetic analysis showed that most (79.3\%) of the Chinese genomes were located in the Indian Ocean lineage (IOL) of the East/Central/South African (ECSA) genotype, and clustered with the strains from India (Fig. 2). Partial strains were located in the Asian genotype and closely related to the strains from Indonesia and Philippines. Besides, the genome sequenced in this study was located in the IOL lineage (Fig. 2), and closely related to a strain (accession no. MF773566) isolated from a patient in Australia who returned from Bangladesh in 2017 and two Chinese strains (accession nos. MG912993 and MH400249) isolated from a local Chikungunya outbreak occurred in Zhejiang Province in 2017 [4].

All of the globally distributed CHIKV genomes were divided into three genotypes, including West Africa (WA), ECSA and Asian genotypes (Fig. 2). Within the ECSA genotype, most genomes (80.9\%) were clustered in the IOL lineage, which was probably originated from Kenya and then spread along Indian Ocean to Comoros, La Reunion, Mauritius, Mayotte, Sri Lanka and India, and then along Indian Subcontinent to Southeast Asia and Europe countries (Fig. 2). Within the Asian genotype, most strains were isolated from the Americas (84.9\%), and developed into a new sublineage named Asian reemerge/Caribbean Islands. This sublineage seems to be originated from Southeast Asia especially Thailand, and spread along the South Pacific to Federated States of Micronesia, Tonga, Samoa, American Samoa, Kiribati, and then to the Americas including Caribbean, United States, Colombia, Dominica, Mexico, Nicaragua, Haidi and so on (Fig. 2).

Subsequently, we characterized the detailed genetic varieties in viral proteins of the CHIKV lineages. A total of 179 distinct sites containing some undescribed sites were identified in the SP and nsP proteins, showing obvious genetic varieties associated with different CHIKV lineages (Fig. 3).

\section{Discussion}

In this study we reported a novel chikungunya case who imported from Myanmar into China. Surprisingly, at least four other cases have been detected to be imported from Myanmar into China in 2019 (http://www.chinanews.com/sh/2019/06-28/8877963.shtml; https://www.cn-

healthcare.com/article/20191106/content-525783. html). Since four chikungunya cases were identified in Myanmyar in 2010, no cases had been documented during 2011-2018 [7]. However, this year health officials in Myanmar have reported a significant increase in chikungunya cases (https://www. mmtimes.com/news/chikungunya-reappears-after-10-years.html). This changing trend indicated the potential reemergence and epidemic of CHIKV in Myanmar.

In order to determine the origin of the Chinese CHIKV strains, we performed a comprehensive phylogenetic analysis. The result indicated that the Chinese strains were mainly sourced from Indian Subcontinent and Southeast Asia, suggesting the potential role for Indian Subcontinent and Southeast 
Asia in promoting the global spread of CHIKV. Then we investigated and observed that two main genotypes probably mediated the recent global spread of CHIKV. The recent emergence and large epidemic of ECSA genotype was first recognized with an outbreak in Kenya in 2004, and then quickly transmitted to Indian Ocean islands and Indian Subcontinent countries and thus named IOL lineage [8]. Phylogenetic analysis revealed the possible transmission route of IOL lineage, suggesting that Indian Subcontinent especially India may be a hub for the rapid global dissemination of the IOL lineage. Currently, the IOL lineage has caused a series of explosive outbreaks involving millions of people in the Indian Subcontinent, Southeast Asia and Europe [2, 3, 9]. The other recent CHIKV reemergence involved the worldwide endemic Asian genotype, which has developed into a new Asian reemerge/Caribbean Islands sublineage. Phylogenetic analysis showed that this sublineage was probably sourced from Southeast Asia, and now endemic in the Americas. The first autochthonous transmission of the Asian genotype in the Americas was reported in 2013 in the Caribbean island. Since then, Caribbean region may become a major dispersal foci for the following dissemination of this new sublineage, which has caused a largescale epidemic, involving almost 45 countries and territories in North, Central and South Americas $[8,10]$. According to the above analyses, we inferred that the recent emergent lineages seemingly displayed different evolutionary paths, and the Indian subcontinent and Southeast Asia regions may act as major hubs for the recent global spread of different CHIKV genotypes.

Previous studies showed that genetic mutations in CHIKV played an important role in the adaptation to new vectors or host immune system [11, 12]. The adaptive mutations in E1 (A226V) and E2 (K252Q) genes among the IOL lineage were demonstrated to be associated with the increased viral fitness towards Ae. albopictus [12], and two specific mutations in E2 (V368A) and 6K (L20M) genes were found in the Caribbean clade, possibly associated with the increased viral fitness and replication towards Ae. aegypti [10]. Surprisingly, here we identified a series of lineage-specific mutations in the SP and nsP proteins, including some undescribed amino acid sites. The mutational hotspots in the SP and nsP genes identified in this study will be particularly informative in determining the viral fitness and tracking the source of the virus.

Notably, we observed the cocirculation of multiple lineages in some regions, especially Southeast Asia, Indian subcontinent and Americas. The cocirculation of Asian and ECSA lineage has occurred in Brazil since 2014, and the similar cocirculation of Asian and IOL lineage was observed in USA [11]. The cocirculation of IOL and Asian lineage appears to be more complicated in Southeast Asia, where there has been identified for years the cocirculation [3]. With increasing globalization, climate changes, the continuing dispersal of Aedes mosquitoes, and adaptive viral mutations, CHIKV will continue to expand its global distribution and cause potential cocirculation of diverse genotypes, becoming an increasing threat to public health.

\section{Conclusion}

In conclusion, here we reported a novel CHIKV isolate from a Chikungunya patient imported from Myanmar into China in 2019. The genome was directly obtained from the serum sample by combined 
MinION sequencing with BGISEQ- 500 sequencing. Phylogenetic analysis showed that the genome sequenced in this study was located in the IOL lineage, and the Chinese strains were mainly sourced from Indian subcontinent and Southeast Asia, implying the potential role of these regions in mediating the transmission of CHIKV. Further analyses indicated that Indian subcontinent and Southeast Asia may act as major hubs for the recent global spread of CHIKV, leading to widespread epidemics and even many large outbreaks. Besides, we identified a series of lineage-specific adaptive mutations possibly associated with vector preference and different transmission routes. Our present study provides better understanding of the recent global evolution of CHIKV, suggesting that CHIKV will pose an increasing threat to public health, and highlighting the urgent need for the strengthened surveillance including the viral diversity surveillance.

\section{Abbreviations}

CHIKV: Chikungunya virus; DENV: Dengue virus; JEV: Japanese encephalitis virus; WNV West Nile virus; YFV: Yellow fever virus. SINV: Sindbis virus; ZIKV: Zika virus; WGS: whole-genome sequencing; ECSA: East/Central/South African; IOL: Indian Ocean lineage; WA: West Africa; CA: Central Africa.

\section{Declarations}

\section{Ethics approval and consent to participate}

Patients signed informed consent forms, and protocols were approved by the institutional review board of the participating units.

\section{Consent for publication}

Not applicable.

\section{Availability of data and material}

The genome sequenced in this study is deposited in GenBank under the accession number MN756625, and the data analyzed during this study are included in Table S1.

\section{Competing interests}

The authors declare that they have no competing interests.

\section{Funding}

This work was supported by grants from the National Science and Technology Major Project (no. 2018ZX10101003), and the National Key R\&D Program of China (no. 2018YFC1603801).

\section{Authors' contributions}


SFQ, HBS and CJW conceived the study. SFQ, JPG, HS, LW, DZA, XCY, XYA, HBS and CJW supervised the work. JPG, XYD, RZH, CJY, HS, LW, DZA, XCY, XYA, and LGW prepared and provided the materials. PHL and PL assembled, annotated, and analyzed the sequence data. SFQ, PHL and PL wrote the draft manuscript, and all authors contributed to and approved the final manuscript.

\section{Acknowledgements}

We thank the Fourth People's Hospital of Zunyi, Guizhou, China for identifying the chikungunya patient and collecting the blood samples in this study.

\section{References}

1. Zeller H, Van Bortel W, Sudre B: Chikungunya: Its History in Africa and Asia and Its Spread to New Regions in 2013-2014. J Infect Dis 2016, 214:S436-S440.

2. Wu D, Wu J, Zhang Q, Zhong H, Ke C, Deng X, Guan D, Li H, Zhang Y, Zhou H, et al: Chikungunya outbreak in Guangdong Province, China, 2010. Emerg Infect Dis 2012, 18:493-495.

3. Rossini G, Landini MP, Sambri V: Evolution and Epidemiology of Chikungunya Virus. Methods Mol Biol 2016, 1426:3-10.

4. Pan J, Fang C, Yan J, Yan H, Zhan B, Sun Y, Liu Y, Mao H, Cao G, Lv L, et al: Chikungunya Fever Outbreak, Zhejiang Province, China, 2017. Emerg Infect Dis 2019, 25:1589-1591.

5. Koren S, Walenz BP, Berlin K, Miller JR, Bergman NH, Phillippy AM: Canu: scalable and accurate longread assembly via adaptive k-mer weighting and repeat separation. Genome Res 2017, 27:722-736.

6. Li D, Liu CM, Luo R, Sadakane K, Lam TW: MEGAHIT: an ultra-fast single-node solution for large and complex metagenomics assembly via succinct de Bruijn graph. Bioinformatics 2015, 31:1674-1676.

7. Tun MM, Thant KZ, Inoue S, Nabeshima T, Aoki K, Kyaw AK, Myint T, Tar T, Maung KT, Hayasaka D, Morita K: Detection of east/central/south African genotype of chikungunya virus in Myanmar, 2010. Emerg Infect Dis 2014, 20:1378-1381.

8. Tanabe ELL, Tanabe ISB, Santos ECD, Marques J, Borges AA, Lima MC, Anderson L, Bassi EJ: Report of East-Central South African Chikungunya virus genotype during the 2016 outbreak in the Alagoas State, Brazil. Rev Inst Med Trop Sao Paulo 2018, 60:e19.

9. Rezza G, Nicoletti L, Angelini R, Romi R, Finarelli AC, Panning M, Cordioli P, Fortuna C, Boros S, Magurano F, et al: Infection with chikungunya virus in Italy: an outbreak in a temperate region. Lancet 2007, 370:1840-1846.

10. Diaz-Quinonez JA, Escobar-Escamilla N, Ortiz-Alcantara J, Vazquez-Pichardo M, de la Luz TorresRodriguez M, Nunez-Leon A, Torres-Longoria B, Lopez-Martinez I, Ruiz-Matus C, Kuri-Morales P, Ramirez-Gonzalez JE: Identification of Asian genotype of chikungunya virus isolated in Mexico. Virus Genes 2016, 52:127-129.

11. Chen R, Puri V, Fedorova N, Lin D, Hari KL, Jain R, Rodas JD, Das SR, Shabman RS, Weaver SC: Comprehensive Genome Scale Phylogenetic Study Provides New Insights on the Global Expansion of 
Chikungunya Virus. J Viro/ 2016, 90:10600-10611.

12. Dutta SK, Bhattacharya T, Tripathi A: Chikungunya virus: genomic microevolution in Eastern India and its in-silico epitope prediction. 3 Biotech 2018, 8:318.

\section{Figures}

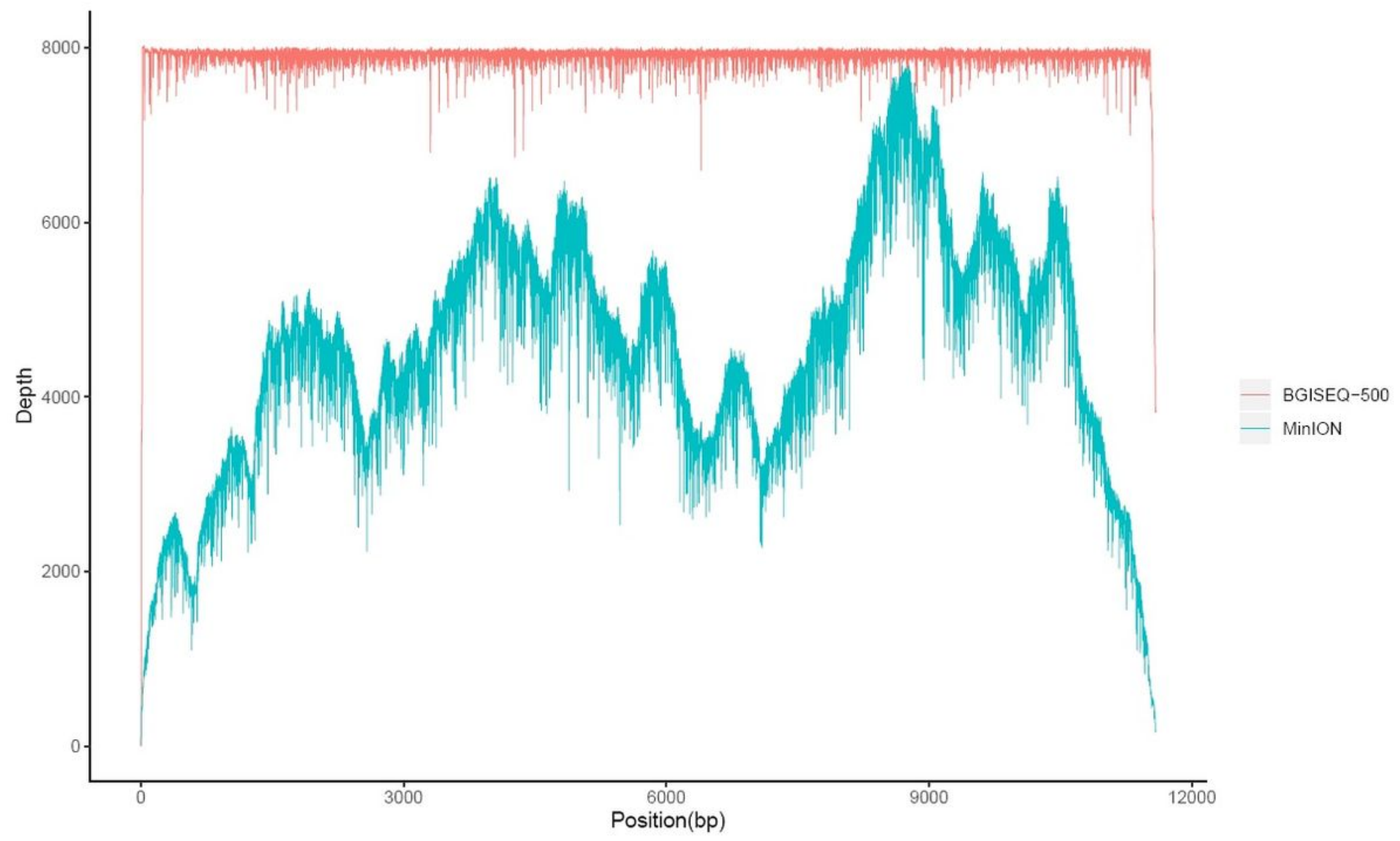

\section{Figure 1}

Coverage depth across the chikungunya viral genome by using MinION and BGISEQ-500 platforms. Coverage depth is shown in blue and red for MinION and BGISEQ-500 platform, respectively. 


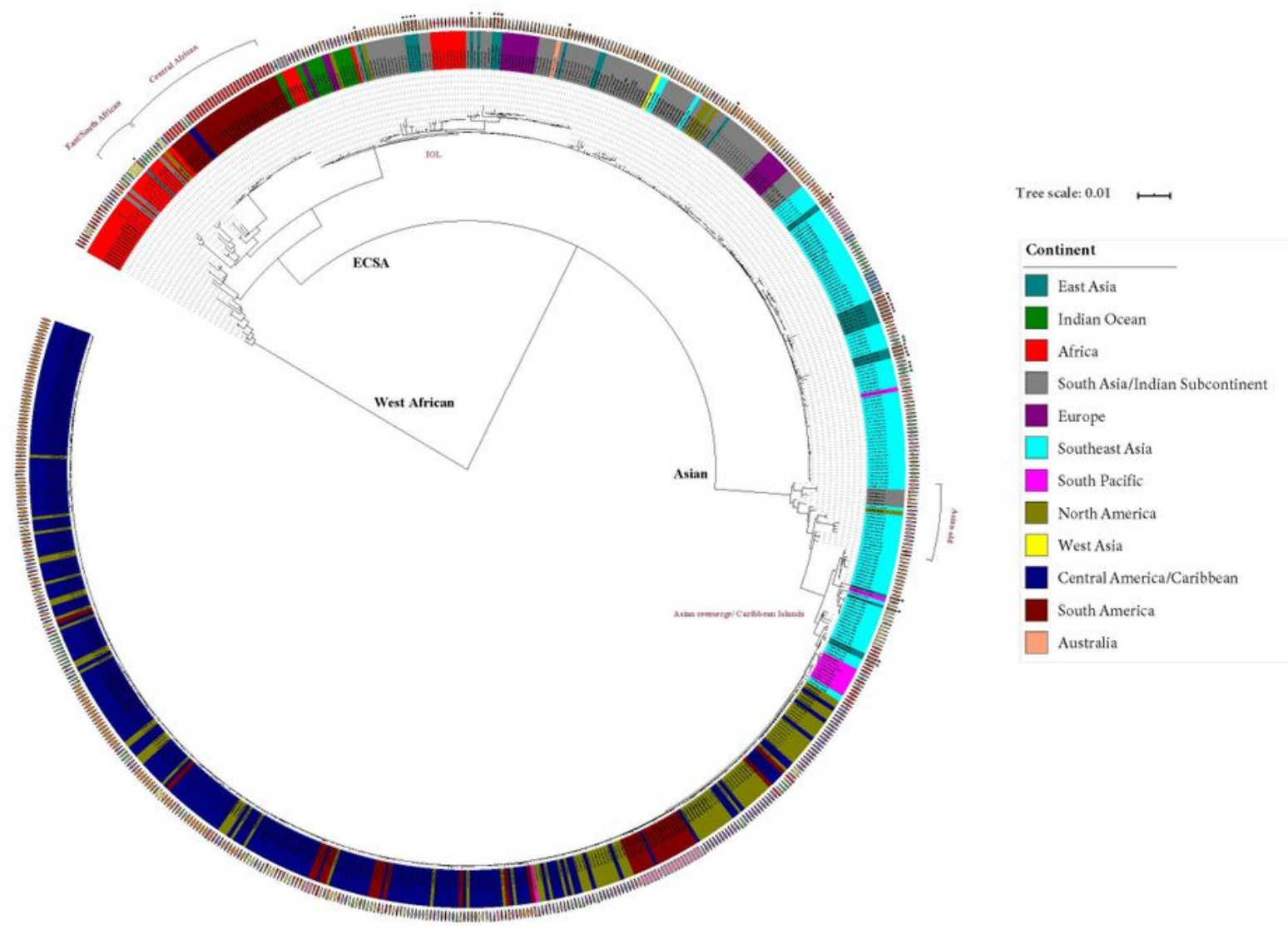

\begin{tabular}{|c|c|c|c|c|}
\hline Country & & & & \\
\hline American somoa & Cayman Islands & Grenada & Madagascar & Panama \\
\hline Angola & Central African Republic & Guadeloupe & Malaysia & Papua New Guinea \\
\hline Anguilla & China & Guatemala & Martinique & Philippines \\
\hline Antigua and Barbuda & Colombia & Haiti & Mauritius & Puerto Rico \\
\hline Australia & Comoros & Honduras & Mayotte & Republic of the Congo \\
\hline Bahamas & Cote d_Ivoire & India & Mexico & Reunion \\
\hline Bangladesh & Cuba & Indonesia & Micronesia & Saint Barts \\
\hline Barbados & Dominica & Italy & Montserrat & Saint Kitts and Nevis \\
\hline Borneo & El Salvador & Jamaica & Myanmar & Saint Lucia \\
\hline Brazil & France & Japan & New Caledonia & Saint Vincent and the Grenadines \\
\hline Cambodia & French Guiana & Kenya & Nicaragua & Samoa \\
\hline Cameroon & French Polynesia & Kiribati & Nigeria & Senegal \\
\hline Caribbean & Gabon & Laos & Pakistan & Seychelles \\
\hline
\end{tabular}

Singapore
South Africa
Sri Lanka
Suriname
Tanzania
Thailand
Tonga
Trinidad and Tobago
Ugrks and Caicos Islands
USA
Venezuela
Virgin Islands
Yemen

\section{Figure 2}

Phylogenetic tree constructed based on maximum likelihood method by using an updated CHIKV genome dataset. A total of 728 genomes are used, including 727 genomes newly retrieved from GenBank and the genome (ZY0814) sequenced in this study. Strains are named by GenBank accession number, sample location and year of isolation, and are color coded for sample location including continents (inner circle) 
or countries (outer circle). The Chinese and Myanmar strains are highlighted in red dot and green triangle, respectively. CHIKV, chikungunya virus; ECSA, East/Central/South African; IOL, Indian Ocean lineage.

A

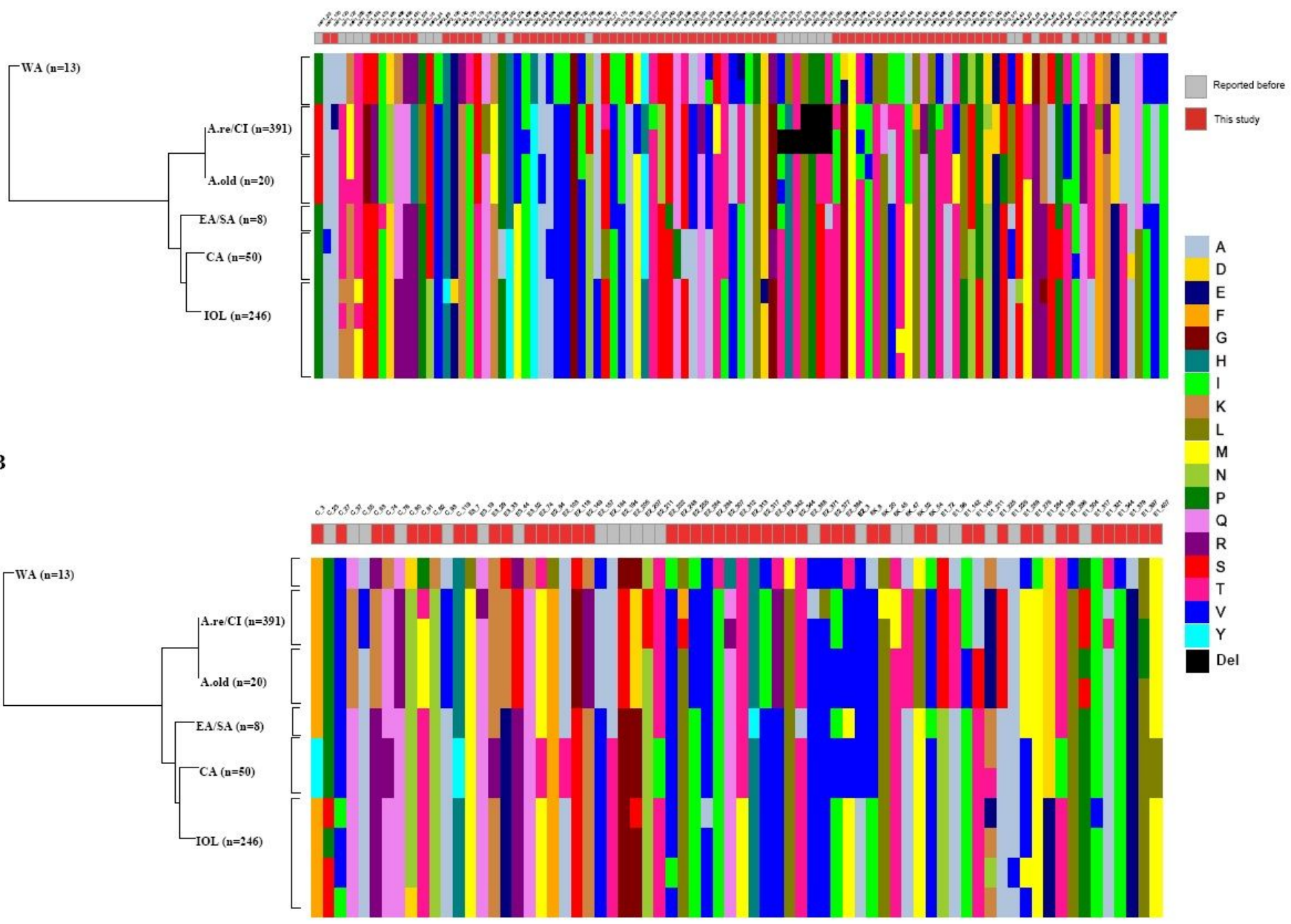

\section{Figure 3}

The lineage-specific genetic varieties in non-structural (panel A) and structural (panel B) proteins of the $\mathrm{CHIKV}$ strains. Alignments of the amino acid sequences were performed to determine the consensus sequence. The consensus sequences from each genotype/lineage were aligned to screen the lineagespecific varieties. The amino acid positions with difference of more than $1 \%$ were selected and the sites unrelated to lineages were removed. There are 72 and 107 mutational sites shown in panel A and panel B, respectively. The amino acid varieties in sites are represented by squares in different colors. The amino acid positions in each viral protein are listed by corresponding numbers. The undescribed mutational sites are marked in red squares and those previously described marked in grey squares. CHIKV, chikungunya virus; WA, West African; A.re/Cl, Asian reemerge/Caribbean Islands; A.old, Asian old; EA/SA, East/South African; CA, Central African; IOL, Indian Ocean lineage. 


\section{Supplementary Files}

This is a list of supplementary files associated with this preprint. Click to download.

- SupplementaryTableS1.xlsx

- SupplementaryTableS2.xlsx 